\begin{tabular}{|c|c|c|}
\hline & Int.J.Curr.Microbiol.App.Sci (2021) 10(09): 284-290 & \\
\hline EXCELLENT & $\begin{array}{l}\text { International Journal of Current Microbiology and Applied Sciences } \\
\text { ISSN: 2319-7706 Volume } 10 \text { Number } 09 \text { (2021) } \\
\text { Journal homepage: http://www.ijcmas.com }\end{array}$ & $\begin{array}{l}\$ 0 \\
\geqslant P\end{array}$ \\
\hline $\begin{array}{l}\text { EXCELLENT } \\
\text { PUBLISHERS }\end{array}$ & & \\
\hline
\end{tabular}

\title{
Evaluation of Genetic Variability of Cryptococcus neoformans VNB Isolates
}

\author{
Luísa Silva Nangi dos Santos ${ }^{1}$, OseiYaw Nyarko ${ }^{2}$ and \\ Leonardo Euripedes de Andrade e Silva ${ }^{2} *$
}
${ }^{1}$ Multiprofessional Health Residency Program, ${ }^{2}$ Department of Infectious Disease, Triangulo Mineiro Federal University, Uberaba, Brazil
*Corresponding author

Keywords

Cryptococcus neoformans, MLST, Cryptococcosis, VNB genotype, phylogenetic analysis

Article Info

Accepted:

15 August 2021

Available Online:

10 September 2021

\section{A B S T R A C T}

Phylogenetic analysis of pathogenic microorganisms contributes to better understanding the distribution of genotypes that may be specific of certain regions and maybe associated with an increased virulence or antifungal resistance. This study performed phylogenetic analysis of 105 Sequence Types (ST) described for the VNB genotype of Cryptococcus neoformans available on the MLST database in order to better understand this population structure. We found three main clusters, being Cluster 1 the largest with 83 STs and Cluster 2 the smallest with three STs. In general, the isolates presented high genetic variability with haplotype diversity (HD) of 0.998 and nucleotide diversity $(\pi$ )of 0.00372 .Results also demonstrated recombination events, although PHY test showed no significance $(\mathrm{p}=5.5511)$. These findings are important since they illustrate that a genotype previously restricted to Africa and now distributed worldwide presents high genetic variability, with potential impact in the development of increased virulence, antifungal resistance, among other factors.

\section{Introduction}

Cryptococcosis is a worldwide distributed fungal infection, and the second most prevalent in HIV/AIDS patients after candidiasis. It may be a localized or disseminated infection, especially in immunocompromised patients (not only due to HIV infection, but also due to diabetes, transplants, hematological neoplasms, and other immunosuppressing factors) and its most severe and prevalent manifestation is acute meningoencephalitis. Immunocompetent patients, however, can also be affected (Mitchell and Perfect 1995, Kurtzman and Fell 2011).The Cryptococcus genus includes several species; however, only Cryptococcus neoformans and Cryptococcus gattii are 
recognized as pathogenic (Kwon-Chung, Polacheck et al., 1982). Cryptococcus neoformans was the first basidiomycete to have its genome thoroughly sequenced (Kavanagh 2006). This species has five main genotypes: VNI, VNII, VNIII, VNIV and VNB. VNB genotype was previously described as a group of serotype A $C$. neoformans, genetically diverse haploid and geographically restricted to Botswana (Chen, Litvintseva et al., 2015). Later on, VNB isolates were described in different parts of the world, such as Sequence Types (ST) restricted to Brazil (Andrade-Silva, Ferreira-Paim et al., 2018).

Different molecular techniques have been used for molecular characterization of Cryptococcus spp. Nowadays, the Multilocus Sequence Typing technique (MLST) is often used in studies of C. neoformans and C. gattii: this method utilizes sequences of different loci to characterize genetic variability of pathogenic microorganisms, being attractive due to its high reproducibility and discriminatory power. The International Society for Human and Animal Mycology (ISHAM) standardized a MLST protocol that is the most widely adopted worldwide. This protocol allows for better comparisons of results to assess the real genetic variability between isolates from different regions of the world (Meyer, Aanensen et al., 2009). Furthermore, its data can be used to determine several factors such as: species recognition, recognition of different populations within species, determining whether a microorganism is purely clonal or has undergone recombination, associating genetic and phenotypic characteristics and assigning the origin of an unknown individual. Therefore, phylogenetic analysis of pathogenic microorganisms contributes to better understanding the distribution of genotypes that may be specific of certain regions and may, sometimes, be associated with an increased virulence or antifungal resistance (Taylor and Fisher 2003).

This study aimed to perform phylogenetic analysis of the described STs for the VNB genotype, obtained according to the MLST consensus for $C$. neoformans, in order to better understanding of the population structure of this genotype.

\section{Materials and Methods}

\section{Fungal sequences evaluated}

We have evaluated all the 103 STs type VNB available at the $C$. neoformans MLST database

(http://mlst.mycologylab.org/Biolomics.aspx? Table=Sequence\%20types\%20C.\%20neoform ans), along with two VNB STs described by the research group from the Mycology Lab at the TrianguloMineiro Federal University (UFTM), Uberaba, Brazil (Ferreira-Paim, Andrade-Silva et al., 2017, Andrade-Silva, Ferreira-Paim et al., 2018), also currently available at MLST database. Multiple alignment was performed in the Clustal $\mathrm{W}$ software

(https://www.ebi.ac.uk/Tools/msa/clustalw2/)( Thompson, Higgins et al., 1994, Thompson, Albert et al., 2014). The phylogenetic analysis was performed in MEGA 6.0 software using the Neighbour Joining (NJ) method with 1,000 bootstrap replicates (Tamura, Stecher et al., 2013). The extent of DNA polymorphisms along with its relevant description parameters, such as the number of polymorphic sites (S), nucleotide diversity (p), number of haplotypes (h), haplotype diversity (Hd), and average number of nucleotide differences $(\mathrm{k})$, were calculated using DNAsp 5.10 (Librado and Rozas 2009). Furthermore, Tajima's D, Fu \& Li's F*, and Fu's Fs tests for neutrality were performed. Recombination degree was calculated using the Watterson estimator (theta) method (also available on DNAsp 
5.10) and by the Splits Tree software, v 4.13.1 (http://www.splitstree.org/) (Barreto de Oliveira, Boekhout et al., 2004, Huson and Bryant 2006). The Pairwise Homoplasy Index (PHY) test was used to infer whether there was statistical significance for recombination.

\section{Results and Discussion}

Among the 105 VNB genotype isolates analyzed, we found three main clusters that were differentiated both via Neighbour Joining (Fig.1) and Split Decomposition (Fig 2) methods. Cluster 1 was the largest with 83 STs. Cluster 2 was composed by 3 STs (ST26, ST393, and ST527) and Cluster 3 by 19 STs. The two VNB isolates from UFTM (STs 527 and ST504) were allocated in Clusters 2 and 3, respectively.

Phylogenetic analysis by the Maximum Likelihood method of the STs available at MLST database for VNB Cryptococcus neoformans

The analysis involved 103 VNB $C$. neoformans isolates from MLST database (mlst.mycologylab.org) and two VNB STs from Uberaba, Brazil (highlighted in red). The phylogenetic tree is drawn to scale, with each branch lengths measuring the number of substitutions per site. Codon positions included were 1 st $+2 n d+3 r d+$ Noncoding. Final dataset presented a total de 4014 positions. Numbers at each branch indicate bootstrap values > 50\% based on 1,000 replicates by the Maximum Likelihood algorithm. The isolates identification is described by their Sequence Type number (ST).

Split decomposition analysis of the $\mathbf{1 0 5}$ VNB Cryptococcus neoformans evaluated

This analysis occurred applying the Neighbour Net algorithm using the uncorrect-p parameter model and evidencing the diversity and branching ambiguities due to recombination events. Isolates are linked to each other via multiple pathways and forming and interconnected network instead of a single forked tree, which suggests the occurrence of recombination; the phi test for recombination implemented in the Split Tree software, however, didn't show significant evidence ( $p$ $=5,5511$ ).

The STs from the three main clusters identified in the phylogenetic analysis (Fig. 1) were also discriminated via split decomposition as follows: Cluster 1: red, Cluster 2: blue, and Cluster 3: green. The two VNB STs from Uberaba are highlighted in red.

Analysis of the DNA polymorphisms is displayed on Table.1. As presented by our data, the isolates showed high genetic variability with a haplotype diversity (HD) of 0.998 and a nucleotide diversity $(\pi)$ of 0.00372 . The results also demonstrated that the isolates had undergone at least 15 recombination events.

In addition, split decomposition analysis demonstrated occurrence of recombination, visualized by the formation of parallelograms between neighbors using the Neighbor Net algorithm (Fig 2). However, PHY test showed no statistically significance for recombination $(p=5.5511)$. The Tajima's D, Fu \& Li's D, Fu \& Li's F, and Fu's F neutrality tests demonstrated evidence of purifying selection or population expansion.

The present study found that the $105 \mathrm{VNB}$ isolates can be sorted in three main clusters (as showed in Fig. 1); this finding corroborates a previous study, performed by Litvintseva et al., (2006), on this serotype, although the clusters were nominated differently (VNBa, $\mathrm{VNBb}$, and $\mathrm{VNBc}$ ) (Litvintseva, Thakur et al., 2006). 
Fig.1 Phylogenetic analysis by the Maximum Likelihood method of the STs available at MLST database for VNB Cryptococcus neoformans

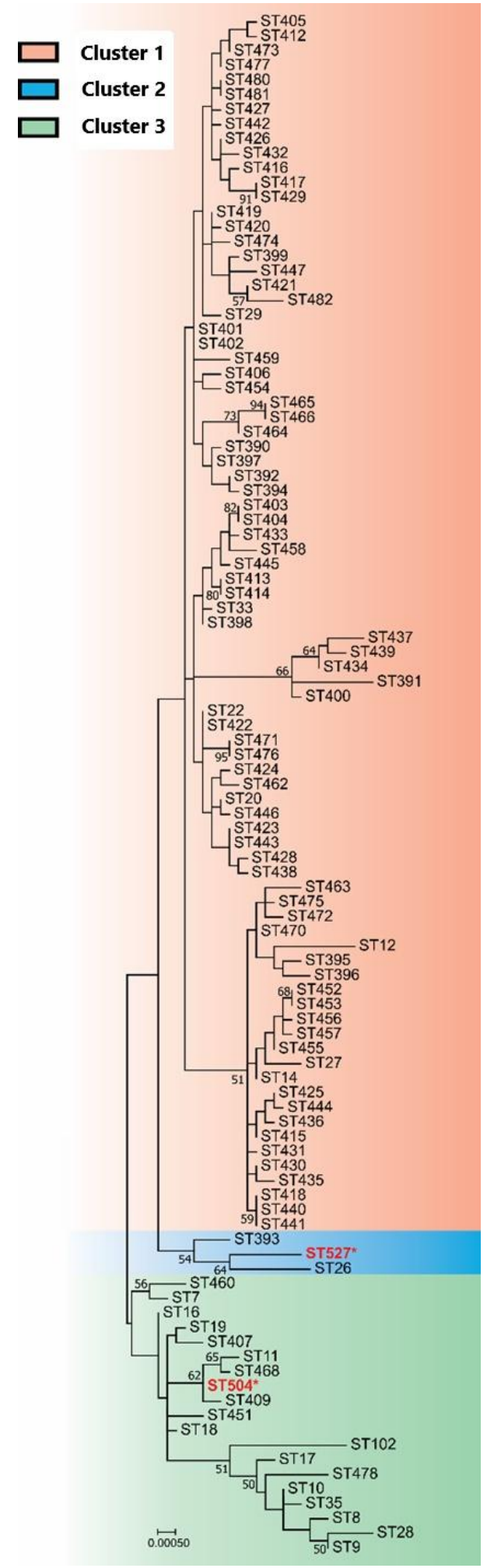


Fig.2 Split decomposition analysis of the 105 VNB Cryptococcus neoformans evaluated

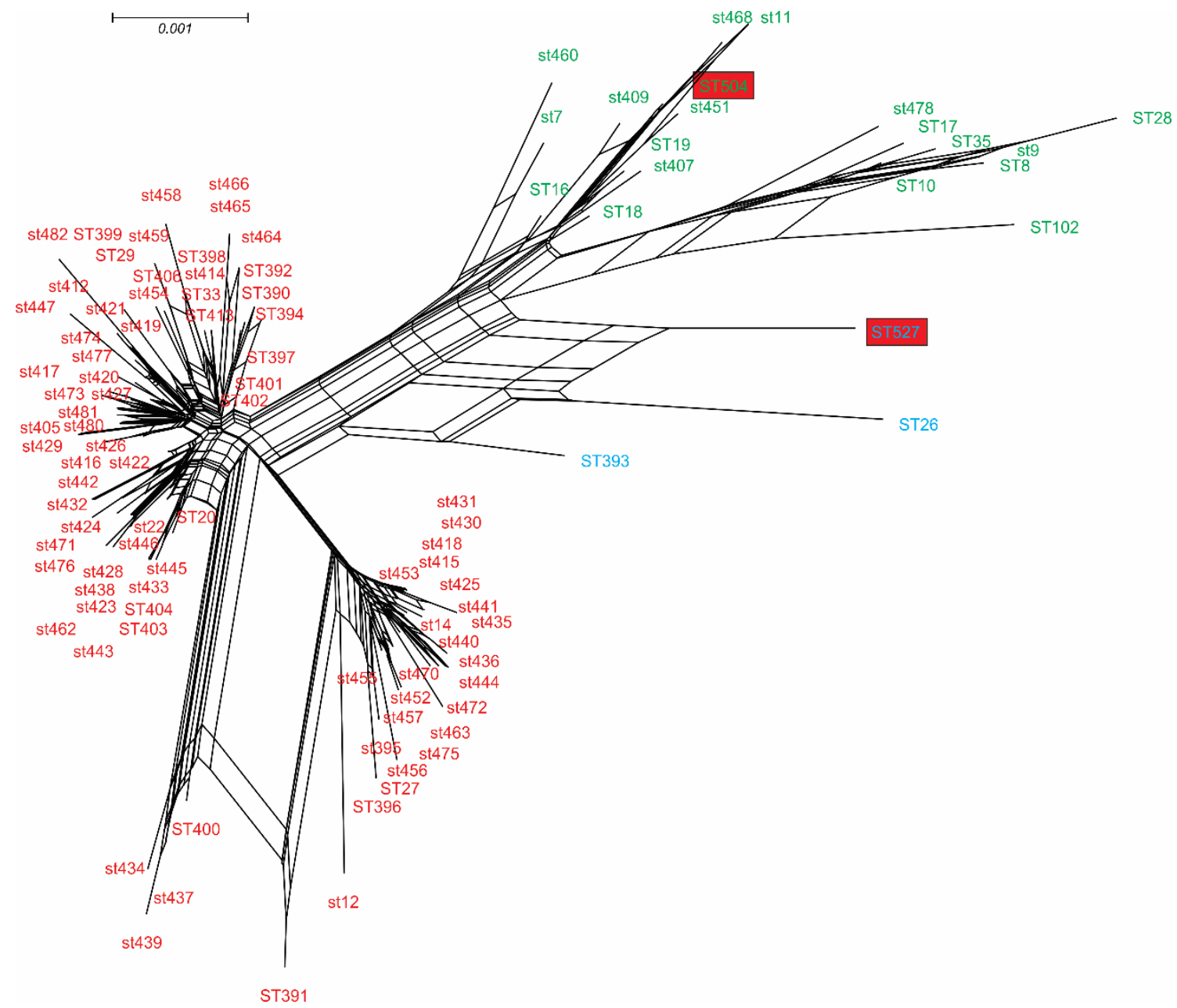

Table.1 DNA polymorphisms in the 105 VNB genotype Cryptococcus neoformans isolates analyzed in this study

\begin{tabular}{|c|c|c|c|c|c|c|c|c|c|c|c|c|c|}
\hline $\begin{array}{c}\text { Number } \\
\text { of isolates }\end{array}$ & Length & $\mathbf{S}$ & $\boldsymbol{\Pi}$ & $\mathbf{k}$ & $\mathbf{h}$ & $\mathbf{H d}$ & $\mathbf{D}$ & $\mathbf{F D}$ & FF & FS & Theta-w & Rm & PHY \\
\hline $\mathbf{1 0 5}$ & 4014 & 95 & 0.00372 & 14.752 & 105 & 0.998 & -0.6417 & -0.37831 & -0.57431 & -94.559 & 18.777 & 15 & 5.5511 \\
\hline
\end{tabular}

Caption: $\mathrm{S}$ - number of polymorphic sites; $\pi$ - nucleotide diversity, Pi; $\mathrm{k}$ - average number of nucleotide differences; $\mathrm{h}$ - number of haplotypes; Hd - haplotype diversity; D - Tajima's D; FD - Fu and Li's D; FF - Fu and Li's F; FS - Fu's Fs; Theta-w - Theta (per sequence) from S; Rm - Minimum number of recombination events.; PHY - Pairwise Homoplasy Index.

The two isolates from Uberaba (ST527 and ST504) were grouped in Clusters 2 and 3, respectively. In a previous study from the research group of the Mycology Lab, they had been clustered in a same group (VNBA and/or VNBII). However, that study analysed significatively less isolates (21 STs) from the
VNB genotype (Andrade-Silva, Ferreira-Paim et al., 2018). Thus, the segregation of the isolates in two different clusters probably occurred due to the incorporation, in the present study, of a larger number of isolates with higher genetic variability among them. 
As for the genetic variability, our findings were higher than described for VNI genotype $C$. neoformans, for which the HDs varied between $0.50-0.65$, and $\pi$ between 0.00120.0033 for isolates from South America; worldwide isolates showed a HD of 0.89 with $\pi=0.0023$ (Litvintseva, Thakur et al., 2006, Chen, Litvintseva et al., 2015, Ferreira-Paim, Andrade-Silva et al., 2017).

Taken together, this study's results demonstrate that the VNB isolates of $C$. neoformans present high genetic variability and occurrence of recombination events. These findings are important since they illustrate that a genotype previously restricted to Africa and now distributed worldwide presents high genetic variability; it potentially results in phenotypical changes that may increase virulence and adaptation to other geographic niches, shift antifungal susceptibility, among other relevant factors.

The use of more data from the isolates such as their clinical and geographic origin, denomination of subgenotypes, susceptibility profile, clinical data from patients can help improving current findings and demonstrating possible correlations between molecular and phenotypic features.

The VNB isolates analyzed in this study were distributed into three main clusters. They displayed high genetic variability and had possibly undergone recombination events.

\section{References}

Andrade-Silva, L. E., K. Ferreira-Paim, T. B. Ferreira, A. Vilas-Boas, D. J. Mora, V. M. Manzato, F. M. Fonseca, K. Buosi, J. Andrade-Silva, B. D. S. Prudente, N. E. Araujo, H. Sales-Campos, M. V. da Silva, V. R. Junior, W. Meyer and M. L. Silva-Vergara (2018). "Genotypic analysis of clinical and environmental
Cryptococcus neoformans isolates from Brazil reveals the presence of VNB isolates and a correlation with biological factors." PLoS One13(3): e0193237.

Barreto de Oliveira, M. T., T. Boekhout, B. Theelen, F. Hagen, F. A. Baroni, M. S. Lazera, K. B. Lengeler, J. Heitman, I. N. Rivera and C. R. Paula (2004). "Cryptococcus neoformans shows a remarkable genotypic diversity in Brazil." J Clin Microbiol42(3): 13561359.

Chen, Y., A. P. Litvintseva, A. E. Frazzitta, M. R. Haverkamp, L. Wang, C. Fang, C. Muthoga, T. G. Mitchell and J. R. Perfect (2015). "Comparative Analyses of Clinical and Environmental Populations of Cryptococcus neoformans in Botswana." Mol Ecol.

Ferreira-Paim, K., L. Andrade-Silva, F. M. Fonseca, T. B. Ferreira, D. J. Mora, J. Andrade-Silva, A. Khan, A. Dao, E. C. Reis, M. T. Almeida, A. Maltos, V. R. Junior, L. Trilles, V. Rickerts, A. Chindamporn, J. E. Sykes, M. Cogliati, K. Nielsen, T. Boekhout, M. Fisher, J. Kwon-Chung, D. M. Engelthaler, M. Lazera, W. Meyer and M. L. SilvaVergara (2017). "MLST-Based Population Genetic Analysis in a Global Context Reveals Clonality amongst Cryptococcus neoformans var. grubii VNI Isolates from HIV Patients in Southeastern Brazil." PLoS Negl Trop Dis11(1): e0005223.

Huson, D. H. and D. Bryant (2006). "Application of phylogenetic networks in evolutionary studies." Mol Biol Evol23(2): 254-267.

Kavanagh, K., Ed. (2006). Medical Mycology: Cellular and Molecular Techniques. Chichester, Jonh Wiley \& Sons Ltd.

Kurtzman, C. P. and J. W. Fell, Eds. (2011). The yeasts: a taxonomic study. 
Amsterdam, The Netherlands, Elsevier.

Kwon-Chung, K. J., I. Polacheck and J. E. Bennett (1982). "Improved diagnostic medium for separation of Cryptococcus neoformans var. neoformans (serotypes A and D) and Cryptococcus neoformans var. gattii (serotypes B and C)." J Clin Microbiol15(3): 535-537.

Librado, P. and J. Rozas (2009). "DnaSP v5: a software for comprehensive analysis of DNA polymorphism data." Bioinformatics25(11): 1451-1452.

Litvintseva, A. P., R. Thakur, R. Vilgalys and T. G. Mitchell (2006). "Multilocus sequence typing reveals three genetic subpopulations of Cryptococcus neoformansvar. grubii (serotype A), including a unique population in Botswana." Genetics172(4): 22232238.

Meyer, W., D. M. Aanensen, T. Boekhout, M. Cogliati, M. R. Diaz, M. C. Esposto, M. Fisher, F. Gilgado, F. Hagen, S. Kaocharoen, A. P. Litvintseva, T. G. Mitchell, S. P. Simwami, L. Trilles, M. A. Viviani and J. Kwon-Chung (2009). "Consensus multi-locus sequence typing scheme for Cryptococcus neoformans and Cryptococcus gattii." Med Mycol47(6): 561-570.
Mitchell, T. G. and J. R. Perfect (1995). "Cryptococcosis in the era of AIDS-100 years after the discovery of Cryptococcus neoformans." Clin Microbiol Rev8(4): 515-548.

Tamura, K., G. Stecher, D. Peterson, A. Filipski and S. Kumar (2013). "MEGA6: Molecular Evolutionary Genetics Analysis version 6.0." Mol Biol Evol30(12): 2725-2729.

Taylor, J. W. and M. C. Fisher (2003). "Fungal multilocus sequence typing-it's not just for bacteria." Curr Opin Microbiol6(4): 351-356.

Thompson, G. R., 3rd, N. Albert, G. Hodge, M. D. Wilson, J. E. Sykes, D. J. Bays, C. Firacative, W. Meyer and D. P. Kontoyiannis (2014). "Phenotypic Differences of Cryptococcus Molecular Types: implications for virulence in a Drosophila model of infection." Infect Immun.

Thompson, J. D., D. G. Higgins and T. J. Gibson (1994). "CLUSTAL W: improving the sensitivity of progressive multiple sequence alignment through sequence weighting, position-specific gap penalties and weight matrix choice." Nucleic Acids Res. 22(22): 4673-4680.

\section{How to cite this article:}

Luísa Silva Nangi dos Santos, Osei Yaw Nyarko and Leonardo Euripedes de Andrade e Silva. 2021. Evaluation of Genetic Variability of Cryptococcus neoformans VNB Isolates. Int.J.Curr.Microbiol.App.Sci. 10(09): 284-290. doi: https://doi.org/10.20546/ijcmas.2021.1009.033 